\title{
Persuasion Artifices To Promote Wellbeing
}

\author{
Fiorella de Rosis ${ }^{1}$, Irene Mazzotta ${ }^{1}$, Maria Miceli $^{2}$, Isabella Poggi $^{3}$ \\ ${ }^{1}$ Intelligent Interfaces, Department of Informatics, University of Bari, \\ \{mazzotta, derosis\}@di.uniba.it \\ ${ }^{2}$ Institute of Cognitive Sciences and Technologies, National Research Council, Roma \\ maria.miceli@istc.cnr.it \\ ${ }^{3}$ Department of Education, University of Roma Tre \\ poggi@uniroma3.it
}

\begin{abstract}
We propose a theory of a-rational persuasion in which we integrate emotional and non emotional strategies by arguing that they both imply reasoning and planning abilities in the two participants. We show some examples of texts from a corpus of persuasion messages in the healthy eating domain and propose a formalism to represent this knowledge. The final goal of our research is to simulate user-adapted persuasion dialogs about healthy eating.
\end{abstract}

\section{Introduction ${ }^{1}$}

Eating habits are an essential component of wellbeing which result of cultural, psychological and educational factors. As such, they consolidate in time and, when wrong, are quite difficult to modify. Information media are masters in employing tricky arguments to persuade the population to consume products of doubtful healthiness. Attempting to contrast this pressure to persuade the population to adopt more appropriate habits by employing only 'rational' and 'scientific' arguments is probably not effective. This is, therefore, one of the domains in which mingling of rational and emotional strategies are justified if not needed. Of course, as suggested by Walton [1], attention should be paid to insure that arguments are relevant and strong: this is a subjective judgement which depends on the persuader's personality and on the context.

In this paper, we propose a method to formalize and represent persuasion strategies as oriented graphs, and show how emotional and non emotional strategies (and also emotional and non emotional components in the same strategy) may interact with, and strengthen, each other. Our theory of a-rational persuasion is outlined in Section 2. A corpus of persuasion messages in the domain of healthy eating is taken as a reference to test our theory and formalism: in Section 3 we describe how we collected this corpus and in Section 4 how we analyzed it. In Section 5, we address the role of uncertainty in persuasion strategies and show how it can be represented in oriented graphs. Section 6 concludes the paper with by briefly pointing to related work.

\footnotetext{
${ }^{1}$ This work was financed, in part, by HUMAINE, the European Human-Machine Interaction Network on Emotion (EC Contract 507422).
} 


\section{Theoretical background}

A relevant issue in the domain of natural argumentation and persuasion is the interaction (synergic or conflicting) between 'rational' or 'cognitive' modes of persuasion and 'irrational' or 'emotional' ones [2]. Miceli et al [3] proposed a model that takes the persuader's P perspective: the model focuses on P's theory of the recipient's $\mathrm{R}$ mind and P's planning strategies for influencing $\mathrm{R}$, that is for changing her mental state so as to make her intend to do a certain action or plan. Our notion of persuasion was circumscribed in relation to further criteria, such as the intentionality of P's persuasive attempt, the use of (verbal or non verbal) communication, and the use of non-coercive forms of influencing. To define a process as persuasion we do not view as necessary that the Recipient is in fact finally persuaded; rather it is necessary that $\mathrm{P}$ has the intention of inducing an intention in $\mathrm{R}$, that $\mathrm{P}$ does so through communication (subliminal persuasion is out of our definition), and does so in a non-coercive way, that is, by letting $\mathrm{R}$ freely conceive that intention or not. Conversely, persuasion can be carried on either by being sincere or through deception, and for either selfish or altruistic purposes; so, it can be either a manipulative or a non manipulative way of influencing people.

We built our theory on Toulmin's theory of argumentation [4] and on further developments [5], in particular Walton's argumentation schemes [6, 7]. A qualifying feature of our model is the attempt to integrate emotional and non emotional persuasion. As its non emotional counterpart, emotional persuasion is aimed at generating, activating or increasing the strength of R's goals, so as to induce in R some intention instrumental to such goals [8]. The specificity of emotional persuasion lies in the means used for accomplishing this task. That is, when using an 'emotional' strategy, P tries to generate, activate, etc. R's goals through the medium of either R's emotions or R's beliefs and goals about her emotions.

Actually, any appeal to emotions is grounded on the strict and manifold relationship between emotions and goals, which is, so to say, 'exploited' by a persuader. At least three kinds of relationship between emotions and goals can be identified. First, emotions signal the destiny (i.e., the possible or actual thwarting or achievement) of our goals, thus accomplishing an informative function about our relationship with the environment (e.g., [9,10]). Second, emotions generate goals. Once an emotion has signalled the achievement or failure of a goal, generally a behavioural response follows, implying the production of some goal (of either the approach or the avoidance type). For instance, fear signals the presence of a danger, and generates the goal to avoid it. Third, emotions 'become' goals: an action may be performed in order (not) to feel a certain emotion. For instance, I may give you a present to feel the joy of making you happy; or do my own duty not to feel guilty. Hence the important role emotions play in learning: an action can be performed (or avoided) not only in view of its outcome, but also in order (not) to feel the associated emotions.

We identify two general modes of emotional persuasion, which are grounded on such relationships between emotions and goals: persuasion through actual arousal of 
emotions and persuasion through appeal to expected emotions. In persuasion through arousal of emotions, the generative relation between emotions and goals is exploited. For instance, P's saying to R 'How disgustingly fat you are!' is meant to provoke R's shame, which should generate R's goal of not losing her face and induce, as a means for this goal, her intention to go on a diet. In persuasion through appeal to expected emotions, the goal-like quality of emotions is exploited, by acting on the recipient's goal (not) to feel a certain emotion. For instance, P's saying to R 'If you are kind to John, you will feel at peace with your conscience (or you will not feel guilty)' is meant to activate R's goal to feel at peace with her conscience (or not to feel guilty), in order to induce in $\mathrm{R}$ the intention to be kind to John as a means for it.

We argue that the rational/irrational, as well as the argumentative/non argumentative dimension, do not allow to distinguish such forms of persuasion from the non emotional ones. In particular, one mode of emotional persuasion, the appeal to expected emotions, can be perfectly rational, as long as 'rational' implies the correct processing of the information available, the derivability of conclusions from premises, and the production of plausible means-ends relationships. An appeal to expected emotions is 'structurally' indistinguishable from any other 'argument from consequences', as defined by Walton ${ }^{2}$ [7]. The only difference resides in the content of the goal on which $\mathrm{P}$ acts: in the appeal to expected emotions, this content is precisely that of 'feeling' a certain emotion rather than having a certain state of the world true. The other mode of emotional persuasion - persuasion through arousal of emotions - works differently from the former, in that the aroused emotion (say, shame) can directly produce a certain goal (say, to save one's face), independent of $\mathrm{R}$ 's reasoning and planning about means-ends relationship. Therefore, this is no doubt a form of non argumentative persuasion. However, in this context 'non argumentative' should be made equal to 'a-rational', rather than 'irrational' (as long as 'irrational' implies going against the dictates of reason). Moreover, it should be stressed that the direct production of a goal through emotional arousal is just one step which is generally included in a more complex persuasion strategy expecting a very 'rational' planning and behaviour on R's part. That is, once a certain goal is emotionally produced, R's reasoning and planning can be called into play in view of its achievement. For instance the goal to save face, together with the belief that being in shape is a means for achieving it, is likely to generate R's goal of being in shape, and the consequent planning as a means for achieving it. Thus, even such a form of persuasion is partially based on the recipient's reasoning and planning abilities, which testifies to the constant mingling and intertwining of rational and a-rational ingredients in most persuasion strategies.

2 Walton's Argument from (Positive or Negative) Consequences is formulated as follows: PREMISE: If A is brought about, then good (bad) consequences will (may plausibly) occur; CONCLUSION: A should (not) be brought about.

(Plus a list of 'critical questions'). As we will see later on, we add to this schema some more conditions, to specify when the conclusion should hold. 


\section{A corpus of persuasion messages about healthy eating}

To assess which artifices are employed by people with no particular competence on healthy eating education, we collected a corpus of persuasion messages with a website (http://www.di.uniba.it/intint/H-persuasion-bi.html). A scenario was presented initially to describe the situation which the subjects participating in the study (the 'persuaders') should imagine to find themselves in:

"Mary, one of your best friends, is a 25 year old girl who follows a wrong diet. She does not eat much fruits and vegetables while tends to overeat meat, sweets and pasta. Try to persuade her to eat more fruits and vegetables and, in doing so, don't forget that Mary is famous for her obstinacy! You know the following facts: eating fruits and vegetables is good for health. They are good sources of vitamin A and $C$, which are important for growth and repair of body tissues, to cleanse the blood and give resistance against colds. Moreover, various epidemiological studies proved that a diet rich in vitamin $A$ and $C$ decreases the risk of coronary heart diseases and stomach cancer. In addition to those facts, consider that health is very important for Mary: she likes sports, undergoes periodical check ups and looks at TV programs about health care. Mary would have enough free time to cook vegetables and delicious fruit dishes. Please, use this information to write a text (from 5 to 10 lines) to argue about your thesis."

The scenario was formulated to suggest the following keypoints in planning a specific strategy:

- Friendship relation between persuader $\mathrm{P}$ and recipient $\mathrm{R}$ (Mary in the previous example)

- R's personality (to be obstinate)

- R's goal (to be in good health, in the previous scenario)

- R's living habits (makes sports, undergoes checkups, looks at specialized TV programs)

- Holding of conditions to do the action $\mathrm{p}$ ( $\mathrm{R}$ has time to prepare vegetables)

- Relationship between desired action (eat vegetables) and likelihood to achieve R's goal.

Cognitive dissonance was implicitly assumed in R's mind. The hypothesis was that, in conditions of cognitive coherence, the intention to perform some action should be a consequence of a set of beliefs, goals and conditions which make the action possible. In the scenario, on the contrary, premises were presumed to be true while the consequence was not. This case of cognitive dissonance was similar to the smoking example originally formulated by Festinger [11]. Four variations of this scenario were displayed randomly to the subject, which differed in two variables: employing positive vs negative arguments and mentioning consequences on health vs appearance. The previous scenario corresponds to the 'positive arguments' and 'mentioning consequences on health' condition. We collected, overall, 33 messages from subjects aged between 23 and 63, of both genders, with various backgrounds. The three variables (age, gender and background) were not statistically associated. In the next Sections we will examine these texts very shortly to single out variations in strategies and to reason on their possible formalization. 


\section{Outline of Corpus Analysis}

We start from the hypothesis that, in conditions of cognitive coherence, the following implication holds: if a given goal is of high value to $R$ and is active in her mind, and $R$ believes that doing a given action implies achieving the goal and that conditions hold to do the action, then $R$ has the intention to do that action.

The main goal (the claim) of a persuasion message is to recommend the activity by strengthening the intention to perform it. This goal may be achieved by combining various techniques which take, as their target, different items in the previous implication:

1) attempt to increase the desirability of the outcome;

2) attempt to convince about activity-outcome relationship;

3) attempt to prove that conditions exist for performing the activity.

In addition, it has been claimed that being aware of a cognitive dissonance between own beliefs and intentions may produce a motivation that results in genuine cognitive changes [11]; therefore, specific reference to inconsistency between the referents' beliefs and goals and their behaviour can also be made to strengthen the persuasiove power of a message. In attempting to produce a motivation based on evoking the cognitive dissonance, the target is the inconsistency between the receivers' set of beliefs and goals and their behaviour.

We analyzed the texts in our corpus by trying to find out whether and how each of these techniques was implemented. Let us start with a first example 3 :

Mary, I believe you should eat more fruits and vegetables.

Aim: recommend the activity.

By making sport, you should know that vegetables are good for health! They strengthen muscles and bones as they are rich in minerals.

Aim: convince about activity-outcome relationship.

The rational strategy adopted is enriched by exploiting evidence about the referent which proves her believing in the relationship ('by making sport, you should know...").

Especially after making sport, a good quantity of fresh season fruit tonifies and rehydrates the body after the big toil!

Same aim and target as in the previous sentence.

Emotional items are introduced in the style ('fresh season fruit', 'big toil': 'faticaccia', in Italian )

Without counting the benefits of vitamins $A$ and $C$ for skin and hair!

Always the same aim and target

Maybe you might get rid of some portion of meat or sweets, to leave more space to fruits and vegetables!

The persuader suggests a plan to implement the activity.

This text is very simple: it is a nearly rational message that we will take as a reference schema in our next analysis. However, very few of the messages in the corpus were formulated according to this schema. This occurred primarily when the subject's background was scientific (computer science in particular, with no gender

3 Translated from Italian: we apologize to our subjects for the bad translation of their very rich texts! 
difference); on the contrary, the majority of subjects with a humanistic background employed more refined and emotional techniques. An example of persuasion through arousal of emotions that we defined in Section 2: by saying "... you pretend you care for your health!, or... a person like you, who cares so much for her health!", the subject aimed at arousing in Mary the emotion of shame by pointing to her incoherent behavior, in order to produce the goal to save face and therefore to care more for her health, as a means to this superordinate goal. Examples of appeal to expected emotions were also frequent. For instance, by saying: "...try to think, Mary, how much more beautiful you might appear and be!" the subject aimed at appealing to Mary's emotional goal to feel attractive. In several cases, the two persuasion techniques were enriched with other a-rational ingredients. For instance:

- persuasion through arousal of emotions was frequently based on evoking explicitly the cognitive dissonance mentioned in the scenario, with the intention to arouse the emotion of shame (and therefore, the goal of saving face) in a Recipient who is (presumably) sensitive to the value of consistency. See the previous examples, and also "...And you, who care so much for being well, you don't think to that?";

- higher-order goals were introduced in the text, such as 'to live in a natural way', 'to satisfy gluttony', 'to enjoy', 'to make friends'. For instance: “...you would contribute to the life of biological peasants, or ...you may always enjoy in preparing gorgeous vegetable meals."

- more or less explicit appeal to emotions was made in some cases: "here is the sagacity of experienced women: you have the creative intelligence on your side... (pride),...I would be delighted to meet you and discuss pleasantly with you... (attraction).

- the recommend the activity section was usually introduced at the beginning of rational texts but only subsequently in less rational ones, after preparing the subject to receive the message. In some cases, this recommendation was substituted with the description of some tempting consequence of the activity: "...tomorrow you invite me at home for dinner and we only eat vegetables and fruits, OK?".

- proofs that conditions existed for making the activity was given, sometimes, in emotional form: "...you, who have time and may enjoy preparing food..." or “....as you have time at your disposal, ...you may find some excellent vegetables and fresh fruits!".

The adoption of various sorts of persuasion techniques proves that our subjects did not consider each of them sufficiently strong per se, and that they attempted to increase the overall effectiveness of the message by combining them appropriately. This is, in our view, an evidence in favour of the hypothesis that the persuasion process cannot be represented with a purely logical formalism but requires to deal with uncertainty of beliefs and strength of goals and arguments. In the following Section, we propose a method to do it. 


\section{Knowledge representation}

We represent persuasion strategies with belief networks (BNs): these are a wellknown formalism to represent probabilistic reasoning in directed acyclic graphs [12] whose nodes represent random (binary or multivalued) variables. The following are some examples of variables denoting statements in the healthy eating domain:

\begin{tabular}{|l|l|}
\hline Implies(GoodApp, SaveFace) & Having a good appearance implies saving face \\
\hline Implies(EatVeg, Health) & Eating vegetables favours good health \\
\hline Tonifies\&Rehydr(Veg, Body) & Vegetables tonify and rehidrate the body \\
\hline Strenghten(Veg,Muscles\&Bones) & Vegetables strengthen muscles and body \\
\hline Benefit(VitA\&C,Skin\&Hair) & Vitamins A and C are of benefit to skin and hair \\
\hline HasBadAspect(Mary) & Mary has a bad aspect \\
\hline MakesSport(Mary) & Mary makes sport \\
\hline MakesCheckUp(Mary) & Mary undergoes frequent checkups \\
\hline LooksAtTVProg(Mary) & Mary looks at TV programs on dieting \\
\hline V-Goal(Mary, Health) & Being in good health is important to Mary \\
\hline V-Goal(Mary, GoodMood) & Being in a good mood is important to Mary \\
\hline A-Goal(Mary, Health) & Being in good health is an active goal in Mary's mind \\
\hline A-Goal(Mary, GoodApp) & Having a good appearance is an active goal in Mary's mind \\
\hline CanDo(Mary, EatVeg) & Conditions exist for Mary to eat vegetables \\
\hline S-Do(Mary, EatVeg) & Mary should have the intention to eat vegetables \\
\hline
\end{tabular}

Oriented arcs in a belief network represent any kind of relationship among variables. In our case, for instance, if 'Mary undergoes frequent checkups', then one may assume, with a certain degree of uncertainty, that 'Being in good health is important to her'. A probability distribution is assigned to the variables associated with the 'root nodes' of the network (those which have no parents) and a conditional probability table to the other nodes. These parameters assign a numerical weight to the relationships among variables, for every combination of their values.

\section{$5.1 \quad$ Node types.}

We introduce a classification of nodes in our BNs which is based on two criteria: aspect of the agent's mental state represented and facts known about the agent:

- intention-node: S-Do(agent, action); agent has the intention to do action;

- valued-goal: V-goal(agent, goal); goal has a high value to agent;

- active-goal: A-goal(agent, goal); goal is active in agent's mind;

- ability-node: CanDo(agent, action); external and internal conditions exist for agent to do action;

- belief-node: (Bel agent $\varphi_{\mathrm{f}}$ ); agent believes that $\varphi_{\mathrm{f}}$;

- evidence-node: an agent feature is true.

Some examples of these nodes may be found in Figures 1 to 5: the meaning of node labels in these figures was defined in the table above. 


\subsection{BN types.}

To represent fragments of argumentation/persuasion strategies, we employ 'elementary belief networks' (EBN) classified according to the type of leaf-node. Some examples:

- 'rational' induction of intentions: this EBN represents the main implication we introduced in Section 3: its leaf node is an intention-node whose parents are a valuedgoal, an active-goal, a belief about the means-end relation and an ability-node. An Argumentation from Consequences scheme represents the relationship among these nodes (see an example in Fig 1).

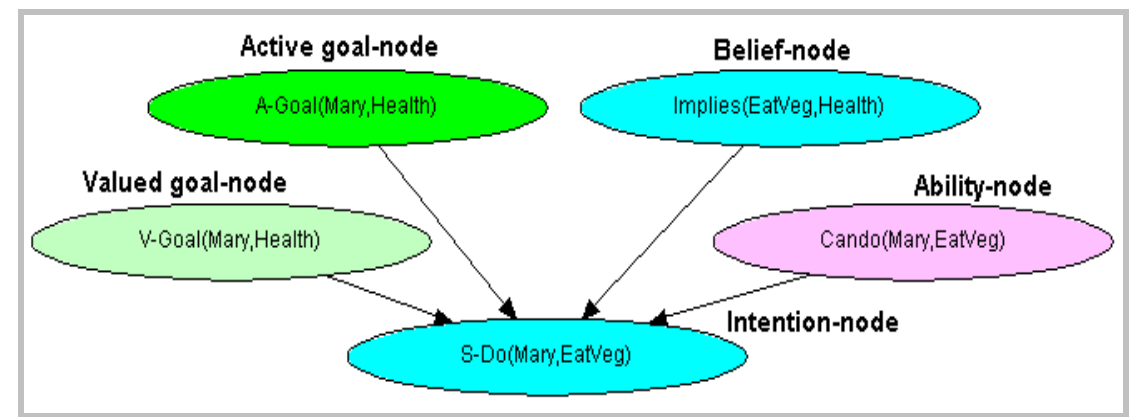

Fig. 1. An intention_EBN in which the goal is 'to be in good health'

- induction of intentions by appeal to the goal to feel emotions: this EBN differs from the previous one only in the type of goal, which is to feel an emotion ('good mood', in the example in Fig 2).

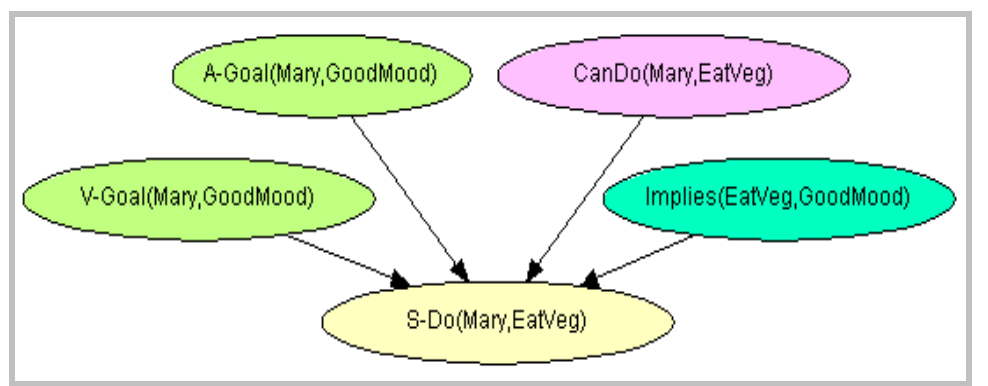

Fig. 2. Another intention_EBN in which the goal is 'too feel in good mood'

- induction of beliefs: here, the leaf node is a belief about a means-end implication (eating vegetables and health, in the example in Fig 3), whose parent nodes are belief or evidence nodes in support of this implication. Various argumentation schemes may represent the relationship among these nodes: for example, Argumentation from Sign [7]. 


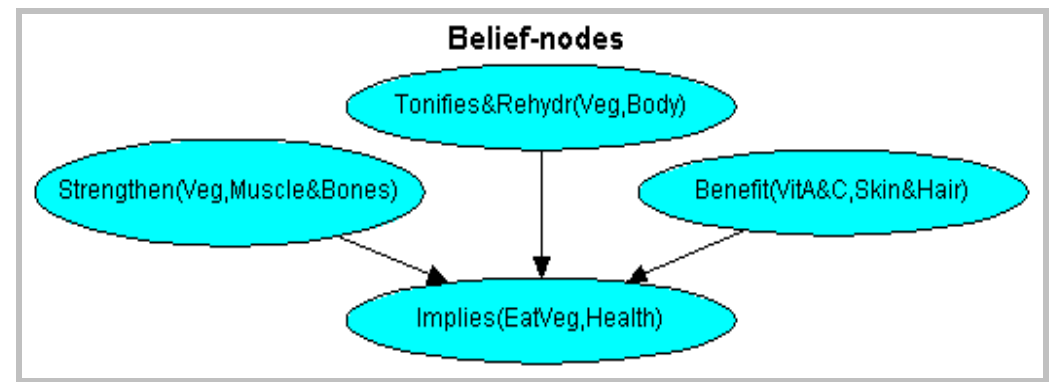

Fig. 3. A belief-EBN in which $\varphi_{\mathrm{f}}$ is 'Eating vegetables favours good health'

- emotional activation of goals: the leaf node is, in this case, an A-Goal node. Its roots represent the emotion-activating belief. In the example in Fig 4the topmost root node is 'to have a bad aspect'; this activates the emotion of 'shame' and, consequently the goal of 'saving face'. Another root node represents the relationship between the final goal of 'having a good appearance' and this superordinate goal.

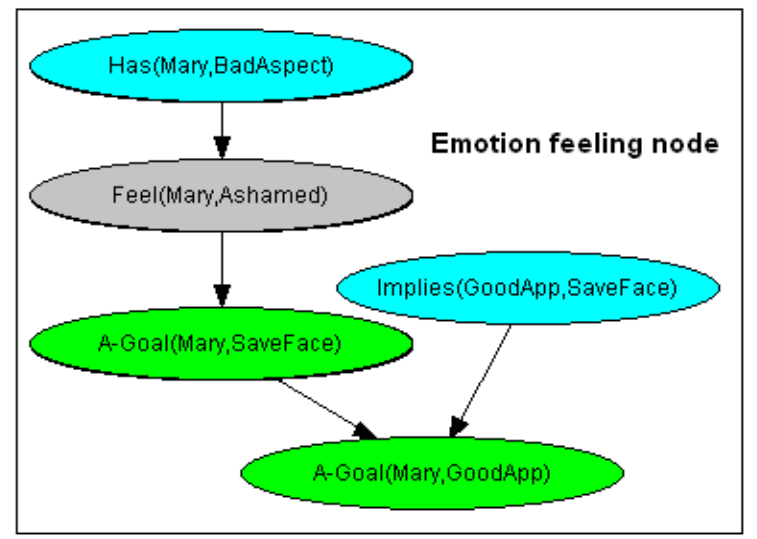

Fig. 4. An active-goal-EBN in which the goal is 'to have a good appearance'

- demonstration of the value of a goal: here, some evidence in support of the assumption that a given goal is of high value to $\mathrm{R}$ is represented (see an example in Fig 5).

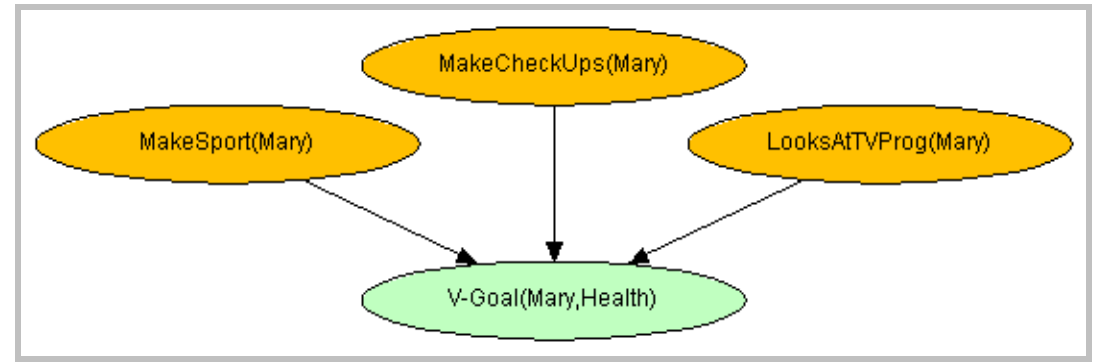

Fig. 5. A valued-goal-EBN (same goal as in figure 4) 


\subsection{Dynamic construction of BNs}

Our BNs are employed to simulate how to plan a persuasion message which is suited to the particular context in which it should be delivered. The BN to employ in a particular context is built starting from an intention-EBN which represents the action the Respondent should be persuaded to perform (for instance, eating vegetables) and a given argumentation scheme (for instance, 'arguing from positive consequences'). This BN may be employed to generate the main part of a monolog or the initial move of a dialog, by applying appropriate natural language generation methods. If, however, the Persuader believes that this elementary message would not be sufficient to persuade the Recipient, other EBNs may be iteratively chained back to it, to strengthen the root-nodes in the network. Chaining back is performed by looking, in the database of EBNs, at the networks which match the node to be strengthened (for instance, a valued-goal node, a CanDo-node, and so on) and at selecting the most appropriate of them in the considered context. The same method may be applied in dialogs to answer 'critical questions' raised by the Recipient.

\section{$5.4 \quad$ An example}

Let us consider again the example text we introduced in Section 3. This text may be represented in a BN which is built by combining an EBN of rational induction of intentions (Fig.1) with other EBNs, of belief induction by means of evidence forwarding (Fig.3) and increase of a goal value (Fig.5). Other combinations may produce texts with more emotional content: for instance, by activating emotionally the goal to be in good health (Fig. 4) or by appealing to the goal to feel an emotion of 'good mood' (Fig.2).

\subsection{How to use the BN}

Belief networks are employed to reason on how coming to know some facts or introducing some hypotheses about the value of nodes in the model, changes the probability of the other variables represented in the model. Probabilistic reasoning on the consequences of evidence about some nodes on the rest of the network may be performed by means of a variety of approximation algorithms, all aimed at reducing the inherent time complexity of the problem. In our case, the algorithm applied was due to Spiegelhalter [13].

We employ our models in a 'what-if' mode, to test the persuasion strength of alternative candidate strategies, given some knowledge about the Recipient R. To this aim, we introduce in the BN the available evidence about user characteristics and the beliefs we might hypothetically induce in the Recipient with our message; we observe the effect of propagating this evidence on the probability of the intentionnode. If this probability is below a given threshold, we label the strategy as 'fully or partially ineffective', we retract the evidence, and start again the process with an alternative strategy. 


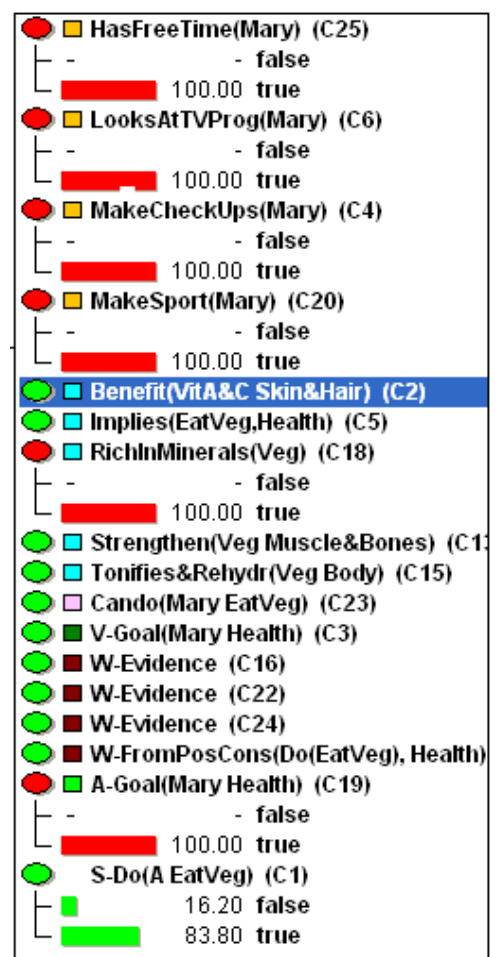

This picture shows an example of 'what-if' kind of reasoning. We chain in a BN the EBNs in Fig 1,2,3,5 and set the parameters so as to simulate a high effectiveness of the rational strategy: the initial probability that Mary will intend to eat vegetables is equal to .36 . We now introduce in the network the knowledge items about Mary which are described in the scenario: has free time, looks at TV programs, makes checkups and makes sport and assume that the goal of being in good health is of high value and active in Mary's mind. We then test the effect of telling Mary that 'vegetables are rich in minerals', and propagate all the mentioned evidence in the network: the probability that Mary will intend to eat vegetables becomes .84. Should of the conditions mentioned above do not hold, this strategy would not be so effective: we might enrich it by activating the goal, either rationally or emotionally. Alternatively, we might test a strategy which is focused on the goal to feel emotions or even combine the two strategies, so as to strengthen their individual effect.

\section{Conclusions}

In this paper we outlined a theory of the possible interaction between emotional and non-emotional persuasion and described how we collected and analyzed a corpus of persuasion texts in the domain of healthy dietary behaviour. With this analysis, we wanted to single out the strategies that were adopted by our subjects in producing a persuasive text. The preliminary results we have got proved that purely rational strategies were employed very infrequently and that emotional elements could be found everywhere, in various forms. Considering probability theory and belief networks as a method for treating uncertainty is not a novelty in the argumentation community. BIAS was the first such system [14]. Gratton [15] proposed to measure the strength of support in probabilistic terms. Das [16] measured probabilistically the confidence that the inference confers to an argument. Green [17] applied Bayesian Networks to describe arguments in medical genetics. Carofiglio [18] discussed how rational strategies may be formalized with this formalism. In this paper, we demonstrated that this formalism enables, as well, to represent the structure of messages which include various kinds of emotional appeals. The main critique that is advanced to reasoning with belief networks concerns the difficulty of estimating their parameters. To improve the reliability of subjective estimates, sensitivity analysis and 
learning from datasets may be applied to single out and learn the most critical parameters. A different problem concerns the last step of message generation, in which the results of reasoning must be translated into natural language texts: rendering the variety and subtlety of language styles we found in our corpus of messages is a problem per se, which merits a separate discussion.

\section{References}

1. Walton, D.N.: The place of emotion in argument. Philadelphia: The Pennsylvania State University Press (1992)

2. Jorgensen, P. E.: Affect, persuasion and communication processes. In Andersen, P. A. and Guerrero L. K. (eds.): Handbook of Communication and Emotion. Academic Press (1998)

3. Miceli, M., de Rosis, F. and Poggi, I.: Emotional and non emotional persuasion. Applied Artificial Intelligence: an International Journal. In press

4. Toulmin, S.: The Use of Arguments. Cambridge, Ma: Cambridge University Press (1958)

5. Kienpointner, M.: How to Classify Arguments. In van Eermeren, F.H., Grootendorst, R., Blair, J.A. and Willard C.A. (eds.): Argumentation Illuminated (1992) 178-188

6. Walton, D.N.: Nonfallacious Arguments From Ignorance. American Philosophical Quarterly 29 (1991) 381-387

7. Walton, D.N., and Reed, C.A.: Argumentation schemes and defeasible inferences. In Carenini, G., Grasso, F., and Reed, C. (eds.): Working Notes of the ECAI'2002 Workshop on Computational Models of Natural Argument, Lyon (2002)

8. Poggi, I.: The goals of persuasion. Pragmatics and Cognition, 13: 2 (2005) 297-336

9. Lazarus, R.S. 1991. Emotion and adaptation. New York: Oxford University Press.

10. Schwarz, N. 1990. Feelings as information: Informational and motivational functions of affective states. In E.T. Higgins and R.M. Sorrentino (Eds.), Handbook of motivation and cognition: Foundations of social behavior. New York: Guilford Press. Vol. 2, pp. 527-561.

11. Festinger, L.: A theory of cognitive dissonance. Evanston, IL. (1957)

12. Pearl, J.: Probabilistic Reasoning in Expert Systems: Networks of Plausible Reasoning. San Mateo, CA: Morgan Kaufmann (Pubs.) (1988)

13. Spiegelhalter, D.J. 1986. Probabilistic Reasoning in L.N.Kanal and J.F.Lemmer (Eds.), Predictive Expert System, Uncertainty in Artificial Intelligence. NorthHolland: Elsevier Science, 4, pp. 357-369.

14. Zukerman, I., Jinah, N., McConachy, R. and George, S.: Recognizing intentions from rejoinders in a bayesian interactive argumentation system. In PRICAI2000 Proceedings, Melbourne, Australia (2000) 241-251

15. Gratton, C.: Counterexamples and degree of support. In Carenini, G., Grasso, F., and Reed, C. (eds.): Proceedings of the Workshop on Computational Models of Natural Arguments, in the scope of ECAI'02 Lyon (2002)

16. Das, S.: Logic of probabilistic arguments. In Carenini, G., Grasso, F., and Reed, C. (eds.): Proceedings of the Workshop on Computational Models of Natural Arguments, in the scope of ECAI'02. Lyon (2002)

17. Green, N.: Towards an empirical model of argumentation in medical genetics. In Carenini, G., Grasso, F., and Reed, C. (eds.): Workshop on Computational Models of Natural Arguments, Acapulco (2003)

18. Carofiglio, V.: Modelling argumentation with belief networks. Workshop on Computational Models of Natural Arguments (CMNA'04), Valencia (2004) 22-24 